\title{
AN INEQUALITY OF TURAN TYPE FOR JACOBI POLYNOMIALS
}

\section{GEORGE GASPER}

Abstract. For Jacobi polynomials $P_{n}^{(\alpha, \beta)}(x), \alpha, \beta>-1$, let

$$
R_{n}(x)=\frac{P_{n}^{(\alpha, \beta)}(x)}{P_{n}^{(\alpha, \beta)}(1)}, \quad \Delta_{n}(x)=R_{n}^{2}(x)-R_{n-1}(x) R_{n+1}(x) .
$$

We prove that

$$
\Delta_{n}(x) \geqq \frac{(\beta-\alpha)(1-x)}{2(n+\alpha+1)(n+\beta)} R_{n}^{2}(x), \quad-1 \leqq x \leqq 1, n \geqq 1,
$$

with equality only for $x= \pm 1$. This shows that the Turán inequality $\Delta_{n}(\alpha) \geqq 0,-1 \leqq x \leqq 1$, holds if and only if $\beta \geqq \alpha>-1$.

1. Let $R_{n}(x ; \alpha, \beta)=P_{n}^{(\alpha, \beta)}(x) / P_{n}^{(\alpha, \beta)}(1)$ where $P_{n}^{(\alpha, \beta)}(x)$ is the Jacobi polynomial [8] of order $(\alpha, \beta), \alpha, \beta>-1$, and let

$$
\Delta_{n}(x ; \alpha, \beta)=R_{n}^{2}(x ; \alpha, \beta)-R_{n-1}(x ; \alpha, \beta) R_{n+1}(x ; \alpha, \beta) .
$$

In [3, p. 153] Karlin and Szegö raised the question of whether or not the Turán inequality

$$
\Delta_{n}(x ; \alpha, \beta)>0, \quad-1<x<1, n \geqq 1,
$$

holds for $\beta \geqq \alpha>-1$. From

$$
\Delta_{n}(-1 ; \alpha, \beta)=\frac{(\beta-\alpha)}{(n+\alpha+1)(n+\beta)}\left(\begin{array}{c}
n+\beta \\
n
\end{array}\right)^{2}\left(\begin{array}{c}
n+\alpha \\
n
\end{array}\right)^{-2}
$$

it is obvious that (1) fails for $\beta<\alpha$. Later [7] Szegö showed that (1) holds for $\beta \geqq|\alpha|, \alpha>-1$, but unfortunately his method fails for the triangle

$$
U=\{(\alpha, \beta): \alpha<\beta<-\alpha,-1<\alpha<0\} .
$$

Recently the author [2] proved (1) for the set

$$
V=\left\{(\alpha, \beta): \beta \geqq \alpha>-1,(\beta-\alpha)(\alpha+\beta)\left(4 \beta^{2}+4 \alpha+1\right) \geqq 0\right\},
$$

Received by the editors July 14, 1971.

AMS 1970 subject classifications. Primary 33A50, 33A65, 42A04; Secondary 33A10, $42 \mathrm{~A} 52$.

Key words and phrases. Inequality, Turán inequality, Jacobi polynomials, ultraspherical polynomials, orthogonal polynomials, Dirichlet kernel.

(c) American Mathematical Society 1972 
which contains most of $U$, but again the method employed failed for the set $U-V$. In this note we give a proof of (1) for the "best possible" range $\beta \geqq \alpha>-1$ and also establish the following stronger result.

ThEOREM. Let $\alpha>-1, \beta>-1$ and $n \geqq 1$. Then

(2) $\Delta_{n}(x ; \alpha, \beta) \geqq \frac{(\beta-\alpha)(1-x)}{2(n+\alpha+1)(n+\beta)} R_{n}^{2}(x ; \alpha, \beta), \quad-1 \leqq x \leqq 1$,

with equality only for $x= \pm 1$.

The method used to prove (2) is a modification of that used by Mukherjee and Nanjundiah [4] for Laguerre and Hermite polynomials, and by Skovgaard [6] for ultraspherical polynomials and Bessel functions. It depends on the observation that if $q_{n}(x)$ is a polynomial of degree $n$ with simple zeros $x_{1}, x_{2}, \cdots, x_{n}$, then

$$
\begin{gathered}
\frac{q_{n}^{\prime}}{q_{n}}=\sum_{k=1}^{n}\left(x-x_{k}\right)^{-1}, \\
-\left(\frac{q_{n}^{\prime}}{q_{n}}\right)^{\prime}=\frac{q_{n}^{\prime 2}-q_{n} q_{n}^{\prime \prime}}{q_{n}^{2}}=\sum_{k=1}^{n}\left(x-x_{k}\right)^{-2},
\end{gathered}
$$

where a prime denotes differentiation with respect to $x$. These identities were used almost a hundred years ago by Laguerre (see [8, p. 120]) to derive bounds for the zeros of the classical polynomials.

Before proving (2), let us first point out some interesting special cases. Using $P_{n}^{(\alpha, \beta)}(-x)=(-1)^{n} P_{n}^{(\beta, \alpha)}(x)$, we find that (2) may be rewritten in the form

$$
\begin{aligned}
& \frac{(n+\alpha)(n+\beta+1)}{(n+\alpha+1)(n+\beta)} R_{n}^{2}(x ; \alpha, \beta)-R_{n-1}(x ; \alpha, \beta) R_{n+1}(x ; \alpha, \beta) \\
& \geqq \frac{(\alpha-\beta)(1+x)}{2(n+\alpha+1)(n+\beta)} R_{n}^{2}(x ; \alpha, \beta), \quad-1 \leqq x \leqq 1,
\end{aligned}
$$

where equality holds only for $x= \pm 1$. Since

$$
R_{n}\left(\cos \theta ; \frac{1}{2},-\frac{1}{2}\right)=\frac{\sin \left(n+\frac{1}{2}\right) \theta}{(2 n+1) \sin (\theta / 2)}
$$

$[8$, p. 60], it follows from (3) that the Dirichlet kernel

$$
D_{n}(\theta)=\frac{1}{2}+\cos \theta+\cdots+\cos n \theta=\frac{\sin \left(n+\frac{1}{2}\right) \theta}{2 \sin (\theta / 2)}
$$


satisfies

$$
D_{n}^{2}(\theta)-D_{n-1}(\theta) D_{n+1}(\theta) \geqq \frac{2(1+\cos \theta)}{(2 n+1)^{2}} D_{n}^{2}(\theta),
$$

with equality only when $\cos \theta= \pm 1$.

If we let $p_{n}(x ; \lambda)=P_{n}^{(\lambda)}(x) / P_{n}^{(\lambda)}(1)$ where $P_{n}^{(\lambda)}(x)$ is the ultraspherical polynomial $\left[8\right.$, p. 81] of order $\lambda, \lambda>-\frac{1}{2}$, then from $[8$, p. 59] we have

$$
\begin{aligned}
p_{2 n}(x ; \lambda) & =R_{n}\left(2 x^{2}-1 ; \lambda-\frac{1}{2},-\frac{1}{2}\right), \\
p_{2 n+1}(x ; \lambda) & =x R_{n}\left(2 x^{2}-1 ; \lambda-\frac{1}{2}, \frac{1}{2}\right) .
\end{aligned}
$$

Hence (2) and (3) yield, for $\lambda>-\frac{1}{2}$ and $-1 \leqq x \leqq 1$,

$$
\begin{gathered}
p_{2 n}^{2}(x ; \lambda)-p_{2 n-2}(x ; \lambda) p_{2 n+2}(x ; \lambda) \geqq-\frac{4 \lambda\left(1-x^{2}\right)}{(2 n-1)(2 n+2 \lambda+1)} p_{2 n}^{2}(x ; \lambda), \\
p_{2 n+1}^{2}(x ; \lambda)-p_{2 n-1}(x ; \lambda) p_{2 n+3}(x ; \lambda) \geqq \frac{4(1-\lambda)\left(1-x^{2}\right)}{(2 n+1)(2 n+2 \lambda+1)} p_{2 n+1}^{2}(x ; \lambda), \\
\frac{(2 n+1)(2 n+2 \lambda-1)}{(2 n-1)(2 n+2 \lambda+1)} p_{2 n}^{2}(x ; \lambda)-p_{2 n-2}(x ; \lambda) p_{2 n+2}(x ; \lambda) \\
\geqq \frac{4 \lambda x^{2}}{(2 n-1)(2 n+2 \lambda+1)} p_{2 n}^{2}(x ; \lambda), \\
\frac{(2 n+3)(2 n+2 \lambda-1)}{(2 n+1)(2 n+2 \lambda+1)} p_{2 n+1}^{2}(x ; \lambda)-p_{2 n-1}(x ; \lambda) p_{2 n+3}(x ; \lambda) \\
\geqq \frac{4(\lambda-1) x^{2}}{(2 n+1)(2 n+2 \lambda+1)} p_{2 n+1}^{2}(x ; \lambda),
\end{gathered}
$$

with equality only for $x=-1,0,1$. This sharpens the inequalities in [ 2 , Corollaries 1 and 2]. For other types of inequalities for orthogonal polynomials see Askey [1] and Patrick [5].

2. In proving the theorem we may assume that $-1<x<1$, for from

$$
R_{n}(1 ; \alpha, \beta)=1, \quad R_{n}(-1 ; \alpha, \beta)=(-1)^{n}\left(\begin{array}{c}
n+\beta \\
n
\end{array}\right)\left(\begin{array}{c}
n+\alpha \\
n
\end{array}\right)^{-1}
$$

it is easy to see that equality holds in (2) when $x= \pm 1$. Since consecutive orthogonal polynomials $q_{n}(x)$ and $q_{n+1}(x)$ cannot have common zeros $[8, \S 3.3]$, we may also assume that $x$ is not a zero of $R_{n}(x ; \alpha, \beta)$. Fix $n \geqq 1, \alpha>-1, \beta>-1$ and let $R_{n}=R_{n}(x ; \alpha, \beta), \Delta_{n}=\Delta_{n}(x ; \alpha, \beta)$. From $[8$, p. 72] we have

$$
R_{n-1}=A_{n} R_{n}^{\prime}+B_{n} R_{n}, \quad R_{n+1}=C_{n} R_{n}^{\prime}+D_{n} R_{n},
$$


where $A_{n}, B_{n}, C_{n}, D_{n}$ are functions of $x$ defined by

$$
\begin{aligned}
& A_{n}=\frac{2 n+\alpha+\beta}{2 n(n+\beta)}\left(1-x^{2}\right), \quad B_{n}=\frac{2 n+\alpha+\beta}{2(n+\beta)}\left(x+\frac{\beta-\alpha}{2 n+\alpha+\beta}\right), \\
& C_{n}=\frac{2 n+\alpha+\beta+2}{2(n+\alpha+1)(n+\alpha+\beta+1)}\left(x^{2}-1\right), \\
& D_{n}=\frac{2 n+\alpha+\beta+2}{2(n+\alpha+1)}\left(x+\frac{\alpha-\beta}{2 n+\alpha+\beta+2}\right) .
\end{aligned}
$$

Using

$$
\left(R_{n}^{\prime} / R_{n}\right)^{\prime}=\left(R_{n} R_{n}^{\prime \prime}-R_{n}^{\prime 2}\right) / R_{n}^{2}
$$

and the differential equation $\left[8\right.$, p. 60] satisfied by $R_{n}$ we obtain

Hence

$$
\begin{aligned}
\left(1-x^{2}\right) R_{n}^{\prime 2}= & {[\alpha-\beta+(\alpha+\beta+2) x] R_{n} R_{n}^{\prime} } \\
& -n(n+\alpha+\beta+1) R_{n}^{2}-\left(1-x^{2}\right)\left(R_{n}^{\prime} / R_{n}\right)^{\prime} R_{n}^{2} .
\end{aligned}
$$

$$
\begin{aligned}
\Delta_{n}= & R_{n}^{2}-\left(A_{n} R_{n}^{\prime}+B_{n} R_{n}\right)\left(C_{n} R_{n}^{\prime}+D_{n} R_{n}\right) \\
= & {\left[1-B_{n} D_{n}+n(n+\alpha+\beta+1)\left(1-x^{2}\right)^{-1} A_{n} C_{n}\right] R_{n}^{2} } \\
& +\left[-A_{n} D_{n}-B_{n} C_{n}+\left(1-x^{2}\right)^{-1}(\beta-\alpha-(\alpha+\beta+2) x) A_{n} C_{n}\right] R_{n} R_{n}^{\prime} \\
& +\left(R_{n}^{\prime} / R_{n}\right)^{\prime} A_{n} C_{n} R_{n}^{2} \\
= & \frac{(\beta-\alpha)(1-x)}{2(n+\alpha+1)(n+\beta)} R_{n}^{2} \\
& -\left[\left(x+\frac{\alpha_{n}^{\prime}}{(2 n+\alpha+\beta)(2 n+\alpha+\beta+2)}-\left(1-x^{2}\right)\left(\frac{R_{n}^{\prime}}{R_{n}}\right)_{n}^{\prime}\right]\right. \\
& \times \frac{A_{n} C_{n} R_{n}^{2}}{1-x^{2}} .
\end{aligned}
$$

Now recall $[8, \S 3.3]$ that all zeros of $R_{n}(x ; \alpha, \beta)$ are real and simple and are located in the open interval $(-1,1)$. Thus

$$
\frac{R_{n}^{\prime}}{R_{n}}=\sum_{k=1}^{n}\left(x-x_{k}\right)^{-1}, \quad\left(\frac{R_{n}^{\prime}}{R_{n}}\right)^{\prime}=-\sum_{k=1}^{n}\left(x-x_{k}\right)^{-2},
$$

where $x_{1}, x_{2}, \cdots, x_{n}$ are the zeros of $R_{n}$, and so

$$
\begin{aligned}
\Delta_{n}= & \frac{(\beta-\alpha)(1-x)}{2(n+\alpha+1)(n+\beta)} R_{n}^{2} \\
& -\frac{\left(1-x^{2}\right)^{-1} A_{n} C_{n} R_{n}^{2}}{(2 n+\alpha+\beta)(2 n+\alpha+\beta+2)} \sum_{k=1}^{n} \frac{E(k, n ; \alpha, \beta)}{\left(x-x_{k}\right)^{2}}
\end{aligned}
$$


with

$$
\begin{aligned}
E(k, n ; \alpha, \beta)= & (2 n+\alpha+\beta)(2 n+\alpha+\beta+2)\left(1-x x_{k}\right) \\
& +\left(\alpha^{2}-\beta^{2}\right)\left(x-x_{k}\right) .
\end{aligned}
$$

To complete the proof it suffices to observe that the expression $-A_{n} C_{n} E(k, n ; \alpha, \beta)$ in (4) is (strictly) positive since $-A_{n} C_{n}>0$ and

$$
\begin{gathered}
\begin{aligned}
E(k, n ; \alpha, \beta)= & 2[2(n-1)(n+\alpha+\beta+2)+2(\alpha+1) \\
& +(\beta+1)(\alpha+\beta+2)]\left(1-x x_{k}\right) \\
& +\left(\alpha^{2}-\beta^{2}\right)(1+x)\left(1-x_{k}\right) \\
= & 2[2(n-1)(n+\alpha+\beta+2)+2(\beta+1) \\
& +(\alpha+1)(\alpha+\beta+2)]\left(1-x x_{k}\right) \\
& +\left(\beta^{2}-\alpha^{2}\right)(1-x)\left(1+x_{k}\right)>0
\end{aligned} \\
\text { for }-1<x, x_{k}<1 .
\end{gathered}
$$

\section{REFERENCES}

1. R. Askey, An inequality for the classical polynomials, Nederl. Akad. Wetensch. Proc. Ser. A 73=Indag. Math. 32 (1970), 22-25. MR 41 \#2079.

2. G. Gasper, On the extension of Turán's inequality to Jacobi polynomials, Duke Math. J. 38 (1971), 415-428.

3. S. Karlin and G. Szegö, On certain determinants whose elements are orthogonal polynomials, J. Analyse Math. 8 (1960/61), 1-157. MR 26 \#539.

4. B. N. Mukherjee and T. S. Nanjundiah, On an inequality relating to Laguerre and Hermite polynomials, Math. Student 19 (1951), 47-48. MR 13, 649.

5. M. L. Patrick, Some inequalities concerning Jacobi polynomials, SIAM J. Math. Anal. 2 (1971), 213-220.

6. H. Skovgaard, On inequalities of the Turán type, Math. Scand. 2 (1954), 65-73. MR 16, 118.

7. G. Szegö, An inequality for Jacobi polynomials, Studies in Math. Anal. and Related Topics, Stanford Univ. Press, Stanford, Calif., 1962, pp. 392-398. MR 26 \#2657.

8. — Orthogonal polynomials, rev. ed., Amer. Math...Soc. Colloq. Publ., vol. 23, Amer. Math. Soc., Providence, R.I., 1959. MR 21 \#5029.

Department of Mathematics, Northwestern University, Evanston, Illinois 60201 